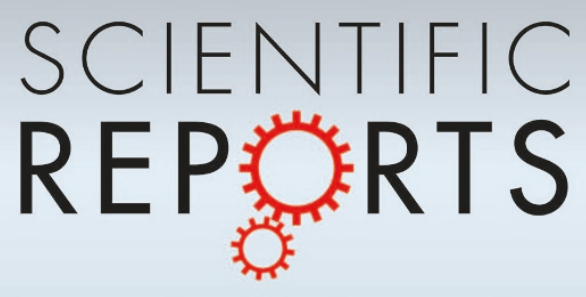

OPEN

SUBJECT AREAS:

DEVELOPMENTAL

BIOLOGY

MORPHOGEN SIGNALLING

Received

10 September 2014

Accepted

17 October 2014

Published

7 November 2014

Correspondence and requests for materials should be addressed to E.E. (eeivers@ calstatela.edu)

* These authors contributed equally to this work.

\title{
Mad linker phosphorylations control the intensity and range of the BMP-activity gradient in developing Drosophila tissues
}

\author{
Abigail Aleman*, Marlyn Rios*, Matthew Juarez, Daniel Lee, Annan Chen \& Edward Eivers
}

Department of Biological Sciences, California State University Los Angeles, 5151 State University Dr., Los Angeles, CA 90032, USA.

The BMP ligand Dpp, operates as a long range morphogen to control many important functions during Drosophila development from tissue patterning to growth. The BMP signal is transduced intracellularly via C-terminal phosphorylation of the BMP transcription factor Mad, which forms an activity gradient in developing embryonic tissues. Here we show that Cyclin dependent kinase 8 and Shaggy phosphorylate three Mad linker serines. We demonstrate that linker phosphorylations control the peak intensity and range of the BMP signal across rapidly developing embryonic tissues. Shaggy knockdown broadened the range of the BMP-activity gradient and increased high threshold target gene expression in the early embryo, while expression of a Mad linker mutant in the wing disc resulted in enhanced levels of C-terminally phosphorylated Mad, a 30\% increase in wing tissue, and elevated BMP target genes. In conclusion, our results describe how Mad linker phosphorylations work to control the peak intensity and range of the BMP signal in rapidly developing Drosophila tissues.

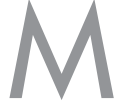
orphogen gradients play an essential role in establishing cell identity during embryonic development, a process which has been found to be evolutionarily conserved. In Drosophila the bone morphogenetic protein (BMP) Decapentaplegic (Dpp), fulfills the criteria of a typical morphogen, where graded amounts of this extracellular ligand have been shown to activate transcription of target genes at different concentration thresholds ${ }^{1-3}$. To activate this signaling cascade, dimers of BMP must first bind to their serine threonine kinase transmembrane receptors which include the type II receptor Punt and type I receptors Thickveins (Tkv) and Saxophone (Sax) $)^{4,5}$. BMP dimer binding to their receptors then causes receptor phosphorylation of the C-terminal domain (-SVS) of the BMP transcription factor Mad. BMP receptor phosphorylated Mad (pMad ${ }^{\text {Cter }}$ ) goes on to form a complex with its common mediator Smad (co-Smad) Medea, translocates and accumulates in the nucleus to activate or repress gene transcription ${ }^{3-6}$. In developing Drosophila tissues, the BMP activity gradient can be identified by visualizing C-terminally phosphorylated Mad intensity levels using a phospho-specific Mad antibody (pMad $\left.{ }^{\mathrm{Cter}}\right)^{7}$. This reagent has revealed that in the blastoderm Drosophila embryo pMad ${ }^{\text {Cter }}$ localizes intensely to about five to seven cell diameters along the dorsal midline, and then phosphorylation sharply drops off to undetectable levels in more lateral regions over a further two to three cell distances ${ }^{8-12}$. In the larval third instar wing imaginal disc, $\mathrm{pMad}^{\mathrm{Cter}}$ levels in the posterior compartment are highest near the anterior/posterior $(\mathrm{A} / \mathrm{P})$ boundary and decline rapidly within a short distance ${ }^{13}$. While in the anterior compartment pMad ${ }^{\text {Cter }}$ levels are extremely low in Dpp expressing cells and higher in cells close to the Dpp source forming a broad peak and steep gradient ${ }^{13}$. A vast array of extracellular modulators help establish graded patterns of Cterminally phosphorylated $\mathrm{Mad}^{14-19}$, and cells within this signaling range must constantly interpret and respond to the intensity of extracellular BMP molecules to determine their cell fate throughout development. Inside the cell a number of mechanisms have been shown to regulate BMP signaling, recent findings have demonstrated that human Smad1 (the vertebrate homolog of Drosophila Mad) linker phosphorylations carried out by mitogen activated protein kinases (MAPKs), cyclin dependent kinases (Cdks) and glycogen synthase kinase 3 (GSK3) are involved in terminating the BMP signal by causing Smad1 to be polyubquitinylated and degraded by the proteasome ${ }^{20-24}$, while phosphatases have been shown to dephosphorylate phosphorylated Smad1 protein $\mathrm{s}^{25-27}$.

This investigation set out to continue our studies into understanding the role Mad linker phosphorylations have in regulating BMP signaling during Drosophila development. Previously, we demonstrated that Mad phospho-resistant linker mutants (serine to alanine mutations, Mad-A212 or MadA204/08) caused hyperactive BMP signaling ${ }^{28}$. This was demonstrated in the Drosophila wing where overexpression of Mad linker mutants 
induced ectopic vein and cross vein tissue, while in Xenopus embryos microinjection of Mad linker mRNAs drastically increased the BMP target gene sizzled and caused strong embryonic ventralization ${ }^{28}$. A role for linker phosphorylations in regulating BMP signals was further supported when immunostainings using antibodies against phospho-serine 212 and phospho-serines 204/08 revealed they required and tracked Mad phosphorylated in its C-terminal domain $\left(\mathrm{pMad}^{\mathrm{Cter}}\right)$ in the early Drosophila embryo ${ }^{28}$. However, our previous study which was primarily focused on investigating a BMP-independent role for Mad in Wingless signaling did not experimentally identify the specific kinases which phosphorylate these Mad linker serines in response to BMP signaling or what the consequences of inhibiting linker phosphorylation had on the pMad ${ }^{\text {Cter }}$ activity gradient in developing tissues. Here we investigated the mechanism of how developmentally graded patterns of C-terminally phosphorylated Mad (the BMP activity gradient) are controlled by Mad linker phosphorylations (an inhibitory linker gradient) in Drosophila embryos and larval wing imaginal discs. First, we identified the two kinases which phosphorylate the linker domain of Drosophila Mad using dsRNA in Drosophila S2 cells; we show that phosphorylation of serine 212 was carried out by Cdk8 which then acts as the priming phosphate to allow the subsequent second and third phosphorylations to be carried out by Shaggy (Sgg) at serine 204 and 208. Second, we found that Sgg depletion in cultured cells and in the oocyte resulted in a notable increase in BMP signaling activity and high threshold target genes in the blastoderm embryo, respectively. Third, we found that maternal depletion of Sgg caused a significant broadening of the dorsal pMad $^{\text {Cter }}$ activity gradient in stage 5/6 embryos which corresponded to increased peak BMP signaling. Finally, we show that phospho-resistant forms of Mad linker mutants could expand the $\mathrm{pMad}^{\mathrm{Cter}}$ gradient beyond its normal range in the wing imaginal disc resulting in tissue overgrowth and increased expression of BMP target genes. The results described in this study provide new insights into how the BMP activity gradient which is known to be regulated by a diverse array of modulators is regulated inside the cell by Mad linker phosphorylations. We hypothesize that this cellular mechanism is essential for keeping the BMP signaling pathway in check during rapid Drosophila tissue development, while also allowing for continuous activation of the BMP pathway in responding cells without resulting in signal saturation.

\section{Results}

With many important questions still to be addressed in relation to the role Mad linker phosphorylations play in BMP signal regulation during Drosophila development, we first set out to investigate what kinase phosphorylates Mad at linker serine 212. In our previous study we had assumed MAPK phosphorylated this site in response to BMP signaling solely based on ours and others published findings in vertebrate systems which demonstrated linker serines flanked by prolines in Smads were phosphorylated by MAPK ${ }^{20-23,28}$. Western blot analysis of Rolled (the Drosophila homolog of vertebrate MAPK) dsRNA treated S2 cells compared to untreated cells revealed that knockdown of this kinase had no effect on the phosphorylation state of serine 212 in the presence of a constitutively activeThickveins receptor (used to generate robust BMP signaling, Fig. 1a). Based on our efficient Rolled knockdown and unchanged Mad phosphorylation state we concluded Rolled was not involved in phosphorylating serine 212 during BMP signaling (Fig. 1a), in contrast to the many findings we and others had described for vertebrate Smad1. More recently, Cdks have been shown to phosphorylate the linker domain of human Smad1 ${ }^{24}$. dsRNA knockdown of Drosophila Cdk8 in BMP-activated S2 cells inhibited phosphorylation of serine 212 compared to untreated cells as demonstrated in western blot analysis and immunostained S2 cells (Fig. 1b-f'), while Mad C-terminal phosphorylations were unaltered by Cdk8 knockdown compared to controls (Fig. 1b- $\mathrm{f}^{\prime}$ ). Western blot analysis also revealed that phosphorylation of serines 204 and 208 were reduced in Cdk8 dsRNA treated cells compared to untreated samples (Fig. 1b). The reduction of serine 204/08 phosphorylation in a Cdk8 depleted background further supports our previous finding which demonstrated that phosphorylation of serines 204/08 required 212 phosphorylation to act as a priming site for their subsequent phosphorylation ${ }^{28}$. In conclusion, we demonstrate for the first time that Cdk8 not Rolled a

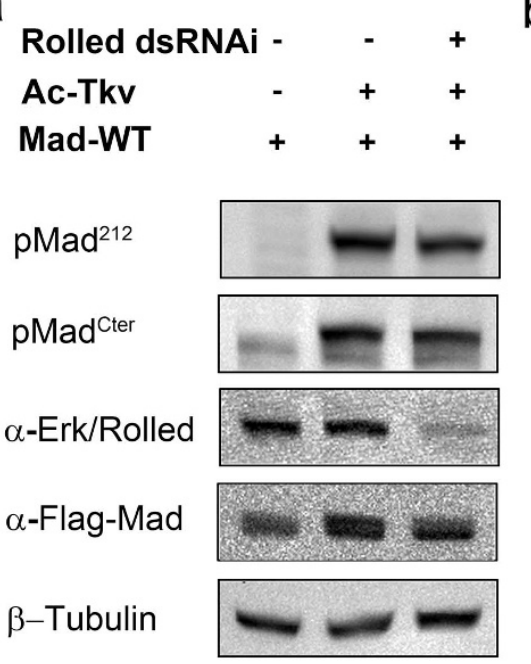

b

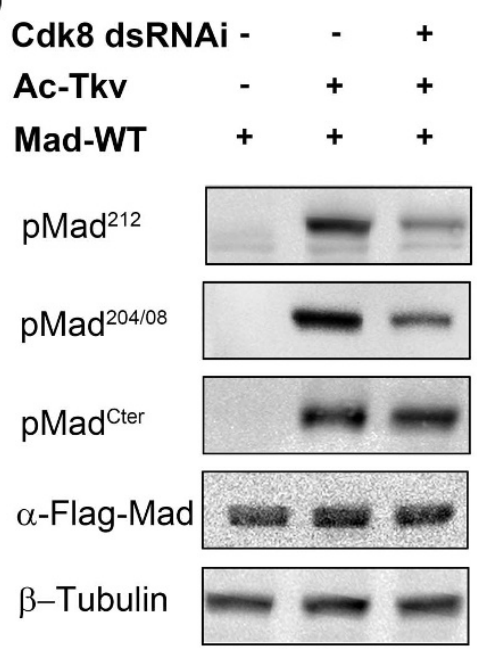

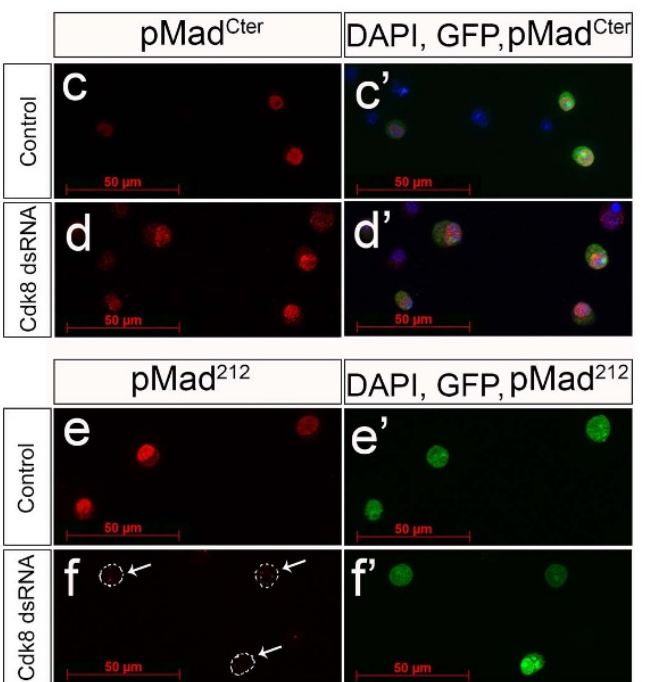

Figure $1 \mid$ Cdk8 phosphorylates serine 212 in the linker domain of Mad in response to active BMP signals. (a) Drosophila S2 cells treated with Rolled dsRNAi show no change in serine 212 or C-terminal phosphorylation levels compared to control untreated cells. Efficiency of Rolled knockdown in dsRNA treated cells demonstrated by the loss of total extracellular signal-related kinase (Erk) protein levels. Loading controls shown by total flag-Mad and $\beta$-tubulin levels. (b) Cdk8 knockdown in S2 cells shows loss of serine 212 phosphorylation compared to control untreated cells. Reduced phosphorylation of serines 204 and 208 in response to loss of serine 212 phosphorylation. Loading controls shown by total flag-Mad and beta-tubulin levels. $\left(\mathrm{c}-\mathrm{c}^{\prime}\right)$ pMad ${ }^{\text {Cter }}$ immunostaining of S2 cells transfected with Mad-WT, activated-Thickveins and GFP. $\left(\mathrm{d}-\mathrm{d}^{\prime}\right)$ pMad ${ }^{\text {Cter }}$ immunostaining is still present in Cdk8 dsRNA treated S2 cells. (e-e') pMad ${ }^{\mathrm{S} 212}$ immunostaining of control untreated S2 cells. $\left(\mathrm{f}-\mathrm{f}^{\prime}\right)$ Loss of pMad ${ }^{\mathrm{S} 212}$ staining in Cdk8 dsRNA treated S2 cells. Cells used for western blot analysis were transfected with pAC-Mad-WT and $+/-$ activated-Thickveins. All western blots were repeated at least 3 times. 
is the kinase that phosphorylates Drosophila Mad at serine 212 during BMP signaling.

The next two serines (204 and 208) we investigated have been shown to be phosphorylated by GSK3 in vertebrate systems ${ }^{22,23}$, thus we analyzed Mad linker phosphorylations in Sgg (the Drosophila homolog of GSK3) depleted S2 cells to confirm that this phosphorylation event in response to BMP signaling is conserved between vertebrate Smads and Drosophila Mad. Knockdown of Sgg using dsRNA revealed loss of serine 204 and 208 phosphorylation, thus demonstrating that this is the phosphorylating kinase for both serines during BMP signaling (Fig. 2a). Phosphorylation of serine 212 was unaffected by Sgg knockdown (Fig. 2a), while Sgg depletion lead to an increase in C-terminally phosphorylated Mad levels (Fig. 2a). To demonstrate the efficiency of our Sgg dsRNA, we show the stabilization of the Wingless pathway protein Armadillo, which is normally degraded in response to Sgg phosphorylation (Fig. $2 \mathrm{a})^{29}$. This is the first direct demonstration that depleting Sgg in S2 cultured cells results in the loss of Mad linker phosphorylation in the presence

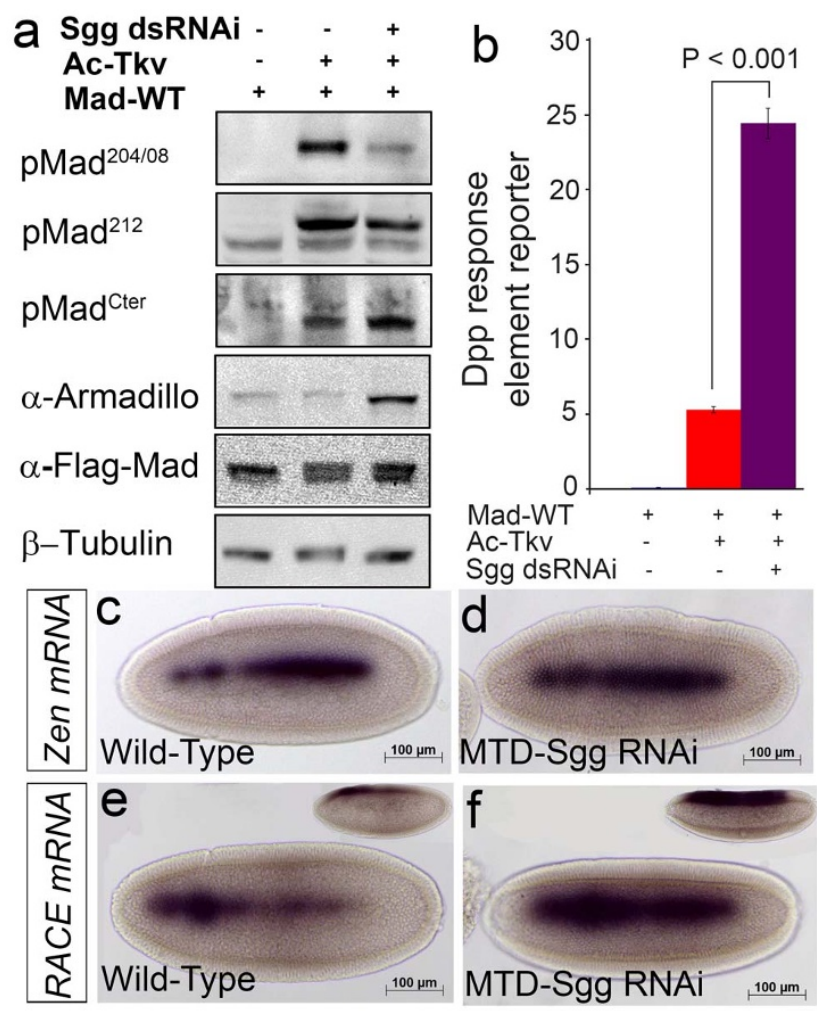

Figure $2 \mid$ Sgg phosphorylation of serines 204 and 208 regulates BMP signaling. (a) Sgg knockdown using dsRNA in Drosophila S2 cells prevented phosphorylation of serines 204 and 208 compared to untreated cells. pMad ${ }^{\text {Cter }}$ levels show a moderate increase in phosphorylation in Sgg knocked down cells compared to untreated controls. Stabilization of Armadillo protein in Sgg treated S2 cells compared to untreated controls demonstrates effectiveness of RNAi knockdown. Armadillo is normally phosphorylated by Sgg resulting in its degradation. Loading controls shown by total flag-Mad and $\beta$-tubulin levels. (b) Fivefold increase in BMP signaling activity measured by a Dpp-response element reporter gene in Sgg dsRNA treated S2 cells compared to untreated controls. (c) Wild type expression of the high threshold BMP target gene Zen in the dorsal most cells of a Drosophila embryo $(\mathrm{n}=34)$. (d) Maternal depletion of Sgg using an inducible RNAi results in increased transcript levels of Zen $(n=20)$. (e) Embryo showing normal wild type expression of the BMP target gene RACE, inset lateral view $(n=35)$. (f) Maternal depletion of Sgg using an inducible RNAi shows a significant increase in RACE transcript levels, inset lateral view $(\mathrm{n}=23)$. All western blots were repeated at least 3 times. Embryos in panels c-f are dorsal views. of active BMP signaling. Analysis of BMP signaling activity using a Dpp response element-luciferase reporter (DRE-Luciferase) ${ }^{30}$ showed that Sgg knockdown resulted in a fivefold increase in signaling in cultured Drosophila S2 cells demonstrating that preventing Sgg phosphorylation of Mad resulted in increased levels of, C-terminally phosphorylated Mad and BMP signaling (Fig. 2a, b).

With the finding that Sgg dsRNA treated S2 cells resulted in loss of linker phosphorylations at serines 204 and 208 and more importantly elevated levels of $\mathrm{pMad}^{\mathrm{Cter}}$, we next investigated the developmental consequences of Sgg depletion on the endogenous BMP phospho-Mad gradient in the early Drosophila embryo. To deplete maternally loaded Sgg from Drosophila embryos, Sgg RNAi was driven in the female germline using the maternal triple driver-Gal4 $\left(\right.$ MTD-Gal4) ${ }^{31,32}$. Analysis of high threshold BMP target genes in Sgg-depleted embryos using whole mount in situ hybridization revealed elevated mRNA transcript levels (Fig. 2c-f). The homeobox gene zerknüllt (Zen) which is normally expressed in a thin stripe in dorsal embryonic cells and requires peak BMP activity was found to have elevated transcript levels in Sgg-depleted embryos (Fig. 2c, d). Zen expression is set by maximal levels of $\mathrm{pMad}^{\mathrm{Cter}}$ and then together in a feed forward mechanism Zen and $\mathrm{pMad}^{\mathrm{Cter}}$ activates angiotensin converting enzyme (Race) expression in the dorsal most cells. Analysis of Race expression in Sgg-depleted embryos revealed strongly increased mRNA levels when compared to controls (Fig. 2e, f) ${ }^{33}$. Finding that high threshold BMP target mRNAs were increased in embryos, we next compared pMad ${ }^{\text {Cter }}$ gradient levels in wild type to Sgg-depleted embryos (Fig. 3a-b). Immunostaining of wild type stage $5 / 6$ embryos with the pMad ${ }^{\text {Cter }}$ antibody revealed typical phospho-expression covering five to seven cell distances in a dorsal nuclear stripe which sharply dropped off over the following two to three lateral cell distances to undetectable levels (Fig. 3a), while in Sgg-depleted embryos a broader intense nuclear stripe of about nine to ten cells distances wide along the embryonic dorsal midline was found (Fig. 3b). Analysis of fluorescent intensity profiles from a $30 \mu \mathrm{m}$ width slice of either wild type or Sgg-depleted embryos confirmed that the $\mathrm{pMad}^{\text {Cter }}$ profiles were broader in range and intensity in Sgg RNAi embryos compared to wild type embryos (Fig. 3c, d, individual intensity profiles in Supplementary information Fig S1). Distance measurements at 200 and 400 intensity units confirmed broader ranges in Sgg-depleted embryos compared to wild type embryos (Fig. 3c, d). Previously, we found that Mad linker phosphorylations tracked C-terminally phosphorylated Mad in the early embryo ${ }^{28}$ (Supplementary information Fig. S2), so we next investigated the consequences of Sgg knockdown on their endogenous phosphorylated expression levels. In agreement with our cultured cell data, we find the absence of linker phosphorylation at serines 204 and 208 in stage 6 Sgg knocked down embryos (Fig. 3e, f). Importantly, we found phospho-serine 212 continued to track Cterminal phosphorylated Mad but now in a similar broader pattern in Sgg-depleted embryos compared to expression in wild type embryos (Fig. 3g, h). The effectiveness of our maternal Sgg knockdown was confirmed by analyzing engrailed protein expression in stage 10 embryos which was found to be expanded to approximately one half the segment width compared to wild type embryos (Fig. 3i, j). This increase in engrailed expression has been previously reported in Sgg genetic null embryos ${ }^{34}$. Our findings demonstrate for the first time a direct link between Sgg phosphorylation of the Mad linker domain and control of the BMP phospho-Mad ${ }^{\text {Cter }}$ gradient in the early Drosophila embryo.

We next turned to the wing imaginal disc to further investigate the role Mad linker phosphorylations have in controlling the BMP activity gradient during development. Hedgehog-Gal4 (HH-Gal4) was used to drive expression of Mad-WT in the posterior wing compartment. This resulted in a mild increase in posterior pMad $^{\text {Cter }}$ intensity levels close to the anterior Dpp ligand source but did not alter, the distance the signal could be detected across the posterior wing pouch 

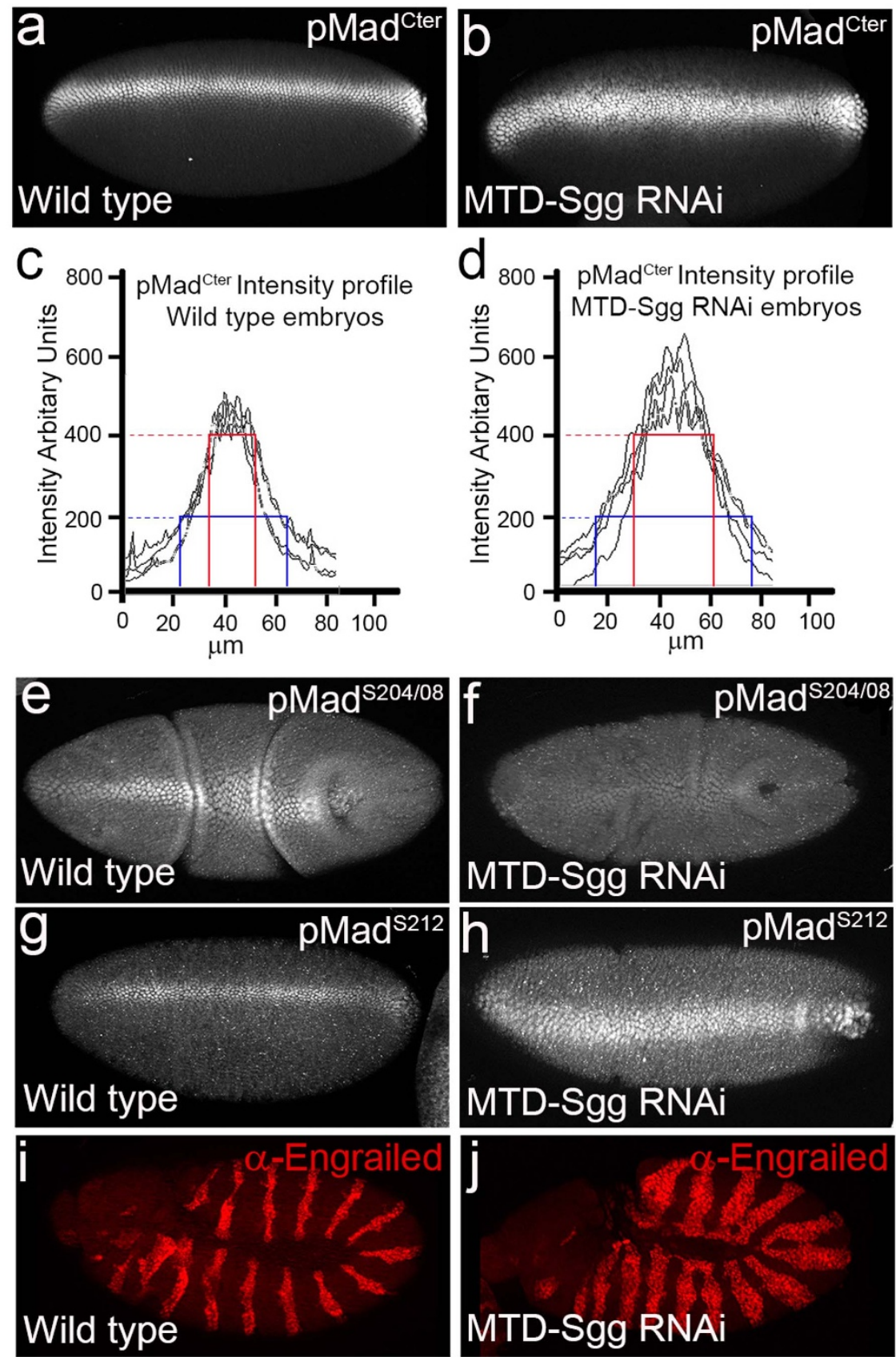

Figure $3 \mid$ Maternal depletion of Sgg results in a broadening of the pMad ${ }^{\text {Cter }}$ activity gradient in the early Drosophila embryo. (a) Expression of Cterminally phosphorylated Mad in a wild type embryo, $\mathrm{n}=29$. (b) Maternal depletion of Sgg using MTD-Gal4 broadened pMad ${ }^{\text {Cter }}$ levels beyond its normal wild type range in the dorsal embryo, $n=23$. (c) Fluorescent intensity profiles measured from 4 separate wild type embryos. Profiles were taken from a $30 \mu \mathrm{m}$ slice perpendicular to the dorsal stripe using Zeiss Apotome software. Intensity levels are arbitrary units on the y-axis and stripe width measured in $\mu \mathrm{m}$ on the x-axis. (d) Fluorescent intensity profiles measured from 4 separate MTD-Sgg RNAi embryos showing an increase in fluorescent intensity levels and overall width of the dorsal stripe. (e) Expression of $\mathrm{pMad}^{\mathrm{S} 204 / 08}$ in a wild type stage 6 embryo, $\mathrm{n}=34$. (f) Loss of pMad ${ }^{\mathrm{s} 204 / 08}$ expression in Sgg depleted embryo, $n=18$. (g) Expression of $\mathrm{pMad}^{\mathrm{s} 212}$ in a wild type embryo, $\mathrm{n}=24$. (h) pMad ${ }^{\mathrm{s} 212}$ shows an increase in the range of phosphorylated expression similar to C-terminally phosphorylated Mad in MTD-Sgg RNAi embryos, $n=19$. (i) Engrailed expression in wild type embryo, $n=50$. (j) Segmental Engrailed expression is increased in Sgg depleted embryos, $\mathrm{n}=56$. Embryos in panels $\mathrm{a}, \mathrm{b}, \mathrm{e}-\mathrm{h}$ are dorsal views and i, $\mathrm{j}$ are lateral views.

(less than $50 \mu \mathrm{m}$ ), or the overall size of the compartment, when compared to wild type discs (Fig. 4a, b'). In contrast, when MadA204/08 was expressed using $\mathrm{HH}-\mathrm{Gal} 4$, enhanced pMad $^{\text {Cter }}$ signal levels abutting the posterior wing compartment boundary were noted and elevated levels of this BMP-activity gradient continued across a broad range of this compartment (beyond $50 \mu \mathrm{m}$ ) in all wing discs analyzed (Fig. 4c, $c^{\prime}$ ). Importantly, pMad ${ }^{\text {Cter }}$ expression levels in the anterior wing compartment were unaffected in HH-Gal4 UAS-
Mad-A204/08 wing discs and anterior pMad $^{\text {Cter }}$ expression levels are not visible in images shown due to the oversaturation of the signal in the posterior wing compartment (Fig. 4c). This demonstrates that posterior overexpression of a Mad linker mutant did not result in any non-cell autonomous effects in the anterior wing imaginal disc. To ensure that the elevated $\mathrm{pMad}^{\text {Cter }}$ levels in posterior wing discs expressing Mad-A204/08 compared to Mad-WT was not due to increased flag-tagged protein levels, Mad-WT and Mad-A204/08 

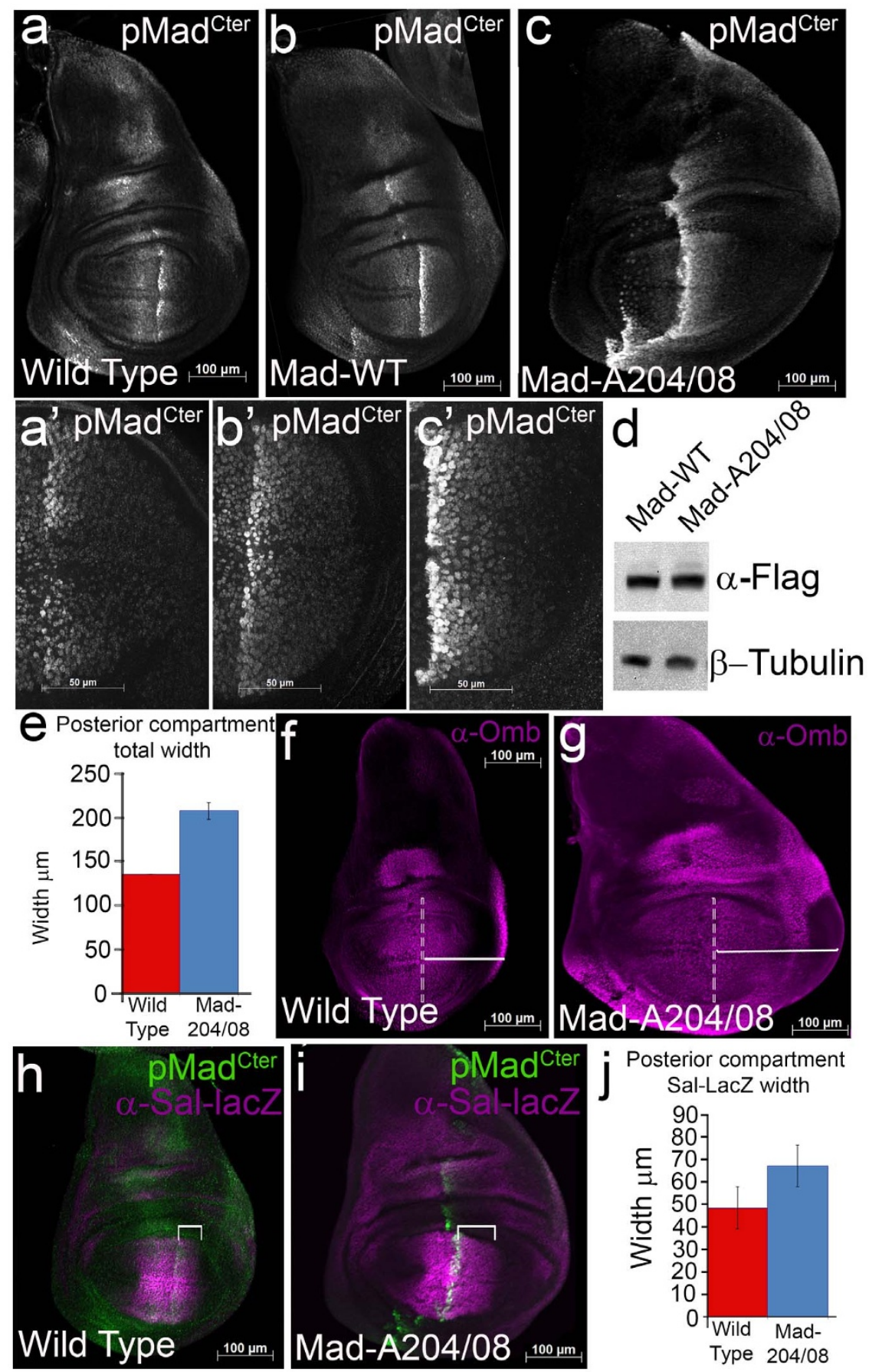

Figure $4 \mid$ Mad linker mutants stabilize the BMP activity gradient in the wing imaginal disc. (a-a') Expression of pMad ${ }^{\text {Cter }}$ in wild type wing imaginal disc, $\mathrm{n}=30$. Anterior $\mathrm{pMad}^{\mathrm{Cter}}$ vertical stripe to the left and posterior vertical pMad ${ }^{\mathrm{Cter}}$ stripe to the right. (b- $\mathrm{b}^{\prime}$ ) Overexpression of Mad-WT in the posterior wing compartment using $\mathrm{HH}-\mathrm{Gal} 4$ causes a slight increase in $\mathrm{pMad}^{\text {Cter }}$ levels, anterior $\mathrm{pMad}^{\text {Cter }}$ expression is unaffected, $\mathrm{n}=35$. $\left(\mathrm{c}-\mathrm{c}^{\prime}\right) \mathrm{Mad}-$ A204/08 misexpression in the posterior wing compartment using HH-Gal4 leads to strong stabilization of the pMad ${ }^{\text {Cter }}$ signal beyond its normal expression range and massive overgrowth of this compartment, $\mathrm{n}=28$. Anterior expression of pMad ${ }^{\text {Cter }}$ is unaffected. (d) Flag-tagged protein levels of Mad-WT and Mad-A208/04 driven using Actin-Gal4 showing equal expression levels and loading. (e) Posterior wing compartment overgrowth was measured to be $30 \%$ larger than wild types discs, wild type discs measured $\mathrm{n}=8$, HH-Gal4;Mad-A204/08 discs measured, $\mathrm{n}=8$. (f) Low threshold BMP target gene Omb protein expression in wild type wing disc, $\mathrm{n}=36$. (g) Increased Omb expression when Mad-A204/08 is overexpressed in the posterior wing compartment using HH-Gal4, $\mathrm{n}=40$. (h) Wild type wing disc showing $\mathrm{pMad}^{\text {Cter }}$ and Sal-lacZ staining, $\mathrm{n}=10$. (i) Misexpression of Mad-A204/08 in the posterior wing compartment using $\mathrm{HH}-\mathrm{Gal} 4$ leads to oversaturated levels of pMad $^{\mathrm{Cter}}$ and an increase in Sal-LacZ expression levels. (j) A slight increase in Sal-LacZ expression range was measured in HH-Gal4; Mad-A204/08 discs $(n=5)$ compared to wild type controls, $\mathrm{n}=5$.

proteins were each driven using Actin-Gal4 and protein levels in first instar larvae were compared using western blotting. Both wild type and linker mutant versions of Mad were found to be expressed at equal levels (Fig. 4d). Mad-A204/08 misexpression using HH-Gal4 was also accompanied by $30 \%$ overgrowth of posterior wing compartment compared to normal wild type discs (Fig. 4e). Previous studies have shown that tissue overgrowth in the wing imaginal disc occurs as a consequence of gain of function for BMP signaling ${ }^{2}$, 
further demonstrating and confirming that inhibition of Mad linker phosphorylation results in hyperactive BMP signals. Next, we examined the BMP target genes Optomotor blind $(\mathrm{Omb})^{35}$ and Spalt major-LacZ (Sal-LacZ) ${ }^{36}$ in the wing imaginal disc when MadA204/08 was driven by HH-Gal4. Misexpression of Mad-A204/08 resulted in an enlarged posterior wing compartment, and elevated Omb (Fig. 4f, g) and Sal-LacZ expression levels (Fig. 4h-i), when compared to control discs. Measurement of the Sal-LacZ expression domain revealed a slight increase in expression range compared to normal wild type discs (Fig. 4j). Finally. misexpression of Mad-A204/ 08 using Apterous-gal4, which drives expression in the dorsal wing compartment (marked by GFP) showed elevated pMad $^{\text {Cter }}$ levels across an increased range in both the dorsal anterior and posterior wing compartments compared to normal $\mathrm{pMad}^{\mathrm{Cter}}$ levels in the ventral wing compartment (Supplementary information Fig 3). These elevated $\mathrm{pMad}^{\text {Cter }}$ levels were found even in the presence of slightly decreased Dpp ligand expression due to a negative feedback loop (Supplementary information Fig 3). Our analysis into Mad linker regulation of the BMP signal supports the vertebrate Smad1 mechanism of action whereby linker phosphorylations are involved in negatively controlling the BMP signal. In our current Drosophila study we add new insights by demonstrating that in the absence of Sgg-induced Mad linker phosphorylations the BMP-activity gradient is strongly increased and operational beyond its normal wild type visual range in developing tissues, resulting in increased gene expression and tissue overgrowth.

\section{Discussion}

In this study we set out to analyze the BMP-activity gradient in the absence of phospho-Mad linker regulation during embryonic and wing imaginal disc development. Our findings demonstrate that linker phosphorylations which mirror the BMP-activity gradient $\left(\mathrm{pMad}^{\text {Cter }}\right)$ were involved in controlling precise $\mathrm{pMad}^{\text {Cter }}$ levels across rapidly dividing tissues. Inhibition of linker phosphorylation resulted in a significant broadening of the BMP activity gradient beyond its normal developmental ranges.

Over the last two decades huge interest has focused on understanding the role vertebrate Smad linker phosphorylations have on BMP signaling output. We and others have shown that inhibition of Smad1 linker phosphorylation could prolong the BMP signal in cultured cells. Briefly the cellular mechanism involved the following, the E3-ligase Smurf1 would bind to linker phosphorylated Smad1, causing its polyubquitinylation and degradation by proteasomes, thus terminating the $\mathrm{BMP}$ signal ${ }^{22,23}$. These vertebrate reports described the mechanism of Smad1 linker regulation but no studies specifically have investigated the consequences of manipulating linker phosphorylation on the BMP-activity gradient (or $\mathrm{pSmad} 1^{\text {Cter }}$ levels) in developing embryonic tissues. In addition, little has been shown in Drosophila except for a previous study where we demonstrated that Mad linker mutants which were phospho-resistant in our serines of interest resulted in hyper activation of the BMP pathway ${ }^{28}$. These conclusions were drawn from a number of assays, first we found overexpressed Mad linker mutants could induce ectopic vein tissue in adult wings and second, Mad mRNAs with linker mutations when microinjected into Xenopus embryos resulted in their strong ventralization, both findings correspond to typical readouts for elevated BMP signaling levels ${ }^{28}$. Here we develop our initial Drosophila findings further by first identifying that both Cdk8 and Sgg phosphorylate three serines in the linker domain of Mad. The finding that Cdk8 phosphorylates serine 212 corrects our initial assumption that MAPK was the phosphorylating kinase and this result is supported by evidence from more recent vertebrate studies which demonstrated that $\mathrm{Cdk} 8$ can phosphorylate serines adjacent to proline residues in human Smad $1^{24}$. The finding that Sgg phosphorylates upstream serines of the initial priming phosphorylation in Drosophila Mad is supported by, and confirms previous reports in vertebrate sys- tems $\mathrm{s}^{22,23}$. Significantly, we present new data showing that Sgg knockdown in cultured S2 cells caused an increase in pMad Cter phosphorylation levels and a fivefold increase in BMP signaling compared to untreated controls. Maternal depletion of Sgg eliminated the negative regulatory effects of Mad linker phosphorylation on the BMP activity gradient resulting in the broadening of the pMad $^{\text {Cter }}$ signal beyond its normal developmental expression range, this was accompanied by elevated levels of high threshold BMP target genes in the early Drosophila embryo. Further analysis of Sgg-depleted embryos revealed the loss of Mad 204/08 linker phospho-gradient, while maintaining serine 212 phosphorylated Mad. Misexpression of phosphorylation insensitive Mad mutants in wing imaginal discs further supported our embryo data showing a strongly stabilized BMP-activity gradient and increased signaling. Our new findings demonstrate a role for Sgg in maintaining precise and sharp pMad ${ }^{\text {Cter }}$ gradient levels during tissue growth and development and links a role for Mad linker phosphorylation in dorsal-ventral patterning in the early embryo.

We hypothesize that linker phosphorylation of Drosophila Mad is involved in terminating the BMP signal via polyubquitinylation and degradation as has been demonstrated for vertebrate Smad $1^{22,23}$. This cellular mechanism is supported by our biochemical and in vivo data we present here, in addition to our previous report that demonstrated overexpressed Mad linker mutants in 293T cells failed to be polyubquitinylated and bind the E3-ligase Drosophila Smurf1 both essential steps in the degradation pathway ${ }^{28}$. This mechanism of degradation would solely be directed at the phosphorylated pool of Mad which represents a tiny amount of the total Mad inside a cell under normal physiological conditions ${ }^{37}$. This explains why we did not see any significant increase stabilization of total flag tagged Mad levels in our western blots.

Mad linker phosphorylations unrelated to BMP signaling have also increasingly been found to be of great importance during signal transduction inside the cell and many novel findings have been uncovered as a consequence. Studies into Mad linker phosphorylations have revealed that linker mutants could mimic gain of function Wingless $\left(\mathrm{Wg}\right.$ ) phenotypes when overexpressed in wing tissue ${ }^{28}$. This finding was followed up by a further study revealing that non-phosphorylated (linker and C-terminal) Drosophila Mad was required for canonical $\mathrm{Wg}$ signaling by interacting with the Pangolin-Armadillo transcriptional complex ${ }^{38}$. Another report found Sgg phosphorylated Mad was involved in a spatially localized round of unconventional Wg signaling during sensory organ development. This study demonstrated that Sgg phosphorylated Mad limited self-renewal of sensory organ precursors ${ }^{39}$. These studies demonstrating required roles for Mad during $\mathrm{Wg}$ signaling are the first to show a BMP-independent role for Mad in cell signaling, in addition to demonstrating that linker phosphorylations were either inhibitory or required for Wg signaling depending on its context and location $^{28,36,37}$. Interestingly, be it BMP or Wg signaling the same kinase, Sgg, phosphorylates serines 208 and 204 showing that even though Mad has evolved unrelated signaling roles inside the cell it has conserved its mechanism of regulating signaling outputs through linker phosphorylations.

We conclude from our findings that the BMP-activity gradient represented by C-terminally phosphorylated Mad is negatively regulated inside the cell by Sgg activity which generates an inhibitory Mad linker phospho-gradient to control the intensity, range and duration of the BMP signal in responding cells.

\section{Methods}

Drosophila strains. Canton S (Bloomington \#1), Sgg RNAi (Bloomington \#35364), Spalt-LacZ (gift from S. Newfeld), Actin-Gal4, Apterous-Gal4, HH-Gal4, MTD-Gal4 (Bloomington \#31777), UAS-MWT-flag, UAS-MadA204/08-flag. All Drosophila crosses were carried out at room temperature $25^{\circ} \mathrm{C}$.

Embryo and wing disc fixation. Drosophila embryos were collected at the desired stage, dechorionated in 50\% Clorox bleach and rinsed thoroughly using distilled $\mathrm{H}_{2} \mathrm{O}$ Embryos were transferred to a glass scintillation vial containing 50\% heptane, 50\% 
PEMFA (PEM and 4\% formaldehyde) solution and gently rocked between 15 and 30 minutes. The lower PEMFA layer was removed and an equal volume of methanol was added to the remaining heptane solution. The vial was then vigorously shaken for 30 seconds and the embryos were allowed to settle to the bottom. The methanol/heptane solution was removed and embryos were washed 3 times with $100 \%$ methanol. Fixed embryos can be stored at this point in $100 \%$ methanol at $-20^{\circ} \mathrm{C}$ for several months. Wing imaginal discs were dissected out of third instar larva in cold Schneider's media. Discs were fixed in Browers solution ${ }^{40}$ for 30 minutes on ice and rinsed using PBS/ $0.02 \%$ Triton X-100.

Antibodies, immunostaining and imaging. Embryos, wing disc and cell immunostainings were carried out using standard protocols. Embryos were immunostained using the following primary antibodies: $\mathrm{pMad}^{212} 1: 500$; $\mathrm{pMad}^{204 / 08}$ $1: 250$; pMad $^{\text {Cter }} 1: 750$; anti-Engrailed (4D9, Hybridoma bank) $1: 10$. Wing discs were immunostained using the following primary antibodies: pMad $^{\mathrm{Cter}} 1: 1000$ (E. Laufer, C. H. Heldin); anti-Omb 1 : 700; $\beta$-Gal (40 1a Hybridoma bank), 1 : 1000; antiFlag rabbit (Sigma) 1:1000; anti-Flag mouse (Sigma) $1: 1000$. The following secondary antibodies (Jackson Laboratories) were used anti-mouse Cy3 conjugated antibody $1: 1000$, anti-mouse 488 conjugated antibody $1: 1000$, anti-rabbit Cy3 conjugated antibody $1: 1000$; anti-rabbit 488 conjugated antibody, $1: 1000$. All tissues were placed in DAPI-containing Vectashield (Vector) and mounted on glass slides. Fluorescent imaging was carried out using a Zeiss Apotome microscope and accompanying Zeiss software (pseudo-coloring). Wing imaginal discs and embryos were imaged at $10 \times$ magnification. Wing pouch images were taken at $20 \times$ magnification. S2 cells were imaged at $63 \times$ magnification.

Fluorescent intensity measurements. Fluorescent intensity profiles were measured from 4 wild type and 4 MTD-Gal4;UAS-Sgg RNAi embryos. Profiles were taken from a $30 \mu \mathrm{m}$ slice perpendicular to the dorsal stripe in each embryo using Zeiss Apotome software. Intensity levels were measured in arbitrary units and stripe width measured in $\mu \mathrm{m}$

In Situ hybridization. In situ hybridization of Drosophila embryos was carried out using standard protocols.

Western blotting. All tissue samples were lysed in RIPA buffer containing phosphatase and protease inhibitors. All western blotting was carried out using standard protocols. Primary antibodies were used at the following concentrations, pMad $^{\text {Cter }}$ (C. H. Heldin, E. Laufer), $1: 1000 ; \mathrm{pMad}^{212}, 1: 4000 ; \mathrm{pMad}^{204 / 08}, 1: 2000$; anti-Armadillo (N2 7A1 Hybridoma bank), $1: 1000$, anti-Erk (cell signaling, $1: 1000$ anti-Flag (Sigma), $1: 1000$, anti- $\beta$-Tubulin (E7-c, Hybridoma bank), $1: 1000$ Secondary antibodies (Thermo Scientific) anti-mouse and anti-rabbit, $1: 1000$

Cell culture, dsRNA treatment and transfection. Drosophila S2 cells were cultured in Schneider's media with 10\% FBS. Growing and culturing of cells followed Drosophila Genomic Resource Center protocols (https://dgrc.cgb.indiana.edu/ Protocols?tab = cells). S2 cells were treated with dsRNA as described in Clemens et $a l^{41}$. S2 cells were transfected with plasmid DNAs 24 hours after dsRNA treatment following the Qiagen Effectene protocol. Luciferase experiments were carried out according to Promega Dual luciferase protocol and readings were measured using a BioTek Synergy 2 reader

1. Nellen, D., Burke, R., Struhl, G. \& Basler, K. Direct and long-range action of a DPP morphogen gradient. Cell. 3, 357-68 (1996).

2. Lecuit, T. et al. Two distinct mechanisms for long-range patterning by Decapentaplegic in the Drosophila wing. Nature. 381, 387-93 (1996).

3. Affolter, M. \& Basler, K. The Decapentaplegic morphogen gradient: from pattern formation to growth regulation. Nat. Rev. Genet. 9, 663-74 (2007).

4. Letsou, A. et al. Drosophila Dpp signaling is mediated by the punt gene product: A dual ligand-binding type II receptor of the TGF $\beta$ receptor family. Cell. 6, 899-908 (1995).

5. Haerry, T. E., Khalsa, O., O'Connor, M. B. \& Wharton, K. A. Synergistic signaling by two BMP ligands through the SAX and TKV receptors controls wing growth and patterning in Drosophila. Development. 20, 3977-87 (1998).

6. Sekelsky, J. J., Newfeld, S. J., Raftery, L. A., Chartoff, E. H. \& Gelbart, W. M. Genetic characterization and cloning of mothers against dpp, a gene required for decapentaplegic function in Drosophila melanogaster. Genetics. 139, 1347-58 (1995)

7. Persson, U. et al. L45 loop in type I receptors for TGF-beta family members is a critical determinant in specifying Smad isoform activation. FEBS Lett. 434, 83-87 (1998).

8. Ross, J. J. et al. Twisted gastrulation is a conserved extracellular BMP antagonist. Nature. 410, 479-83 (2001).

9. Rushlow, C., Colosimo, P. F., Lin, M. C., Xu, M. \& Kirov, N. Transcriptional regulation of the Drosophila gene zen by competing Smad and Brinker inputs. Genes Dev. 15, 340-51 (2001).

10. Dorfman, R. \& Shilo, B. Z. Biphasic activation of the BMP pathway patterns the Drosophila embryonic dorsal region. Development. 6, 965-72 (2001)

11. Sutherland, D. J., Li, M., Liu, X. Q., Stefancsik, R. \& Raftery, L. A. Stepwise formation of a SMAD activity gradient during dorsal-ventral patterning of the Drosophila embryo. Development. 23, 5705-16 (2003).
12. O'Connor, M. B., Umulis, D., Othmer, H. G. \& Blair, S. S. Shaping BMP morphogen gradients in the Drosophila embryo and pupal wing. Development. 133, 183-93 (2006)

13. Tanimoto, H., Itoh, S., ten Dijke, P. \& Tabata, T. Hedgehog creates a gradient of DPP activity in Drosophila wing imaginal discs. Mol. Cell. 1, 59-71 (2000).

14. Marqués, G. et al. Production of a DPP activity gradient in the early Drosophila embryo through the opposing actions of the SOG and TLD proteins. Cell. 91, 417-26 (1997).

15. Nakato, H., Fox, B. \& Selleck, S. B. Dally, a Drosophila member of the glypican family of integral membrane proteoglycans, affects cell cycle progression and morphogenesis via a Cyclin A-mediated process. J. Cell Sci. 115, 123-30 (2002).

16. Fujise, M. et al. Dally regulates Dpp morphogen gradient formation in the Drosophila wing. Development. 130, 1515-22 (2003).

17. Belenkaya, T. Y. et al. Drosophila Dpp morphogen movement is independent of dynamin-mediated endocytosis but regulated by the glypican members of heparan sulfate proteoglycans. Cell. 119, 231-44 (2004).

18. Shimmi, O., Umulis, D., Othmer, H. \& O'Connor, M. B. Facilitated transport of a $\mathrm{Dpp} / \mathrm{Scw}$ heterodimer by Sog/Tsg leads to robust patterning of the Drosophila blastoderm embryo. Cell. 6, 873-86 (2005).

19. Vuilleumier, R. et al. Control of Dpp morphogen signalling by a secreted feedback regulator. Nat. Cell Biol. 6, 611-7 (2010).

20. Kretzschmar, M., Doody, J. \& Massagué, J. Opposing BMP and EGF signaling pathways converge on the TGF- $\beta$ family mediator Smad1. Nature, 389, 618-622 (1997).

21. Pera, E. M., Ikeda, A., Eivers, E. \& De Robertis, E. M. Integration of IGF, FGF and anti-BMP signals via Smad1 phosphorylation in neural induction. Genes Dev. 17, 3023-3028 (2003).

22. Fuentealba, L. C. et al. Integrating patterning signals: Wnt/GSK3 regulates the duration of the BMP/Smad1 signal. Cell. 131, 980-993 (2007).

23. Sapkota, G., Alarcón, C., Spagnoli, F. M., Brivanlou, A. H. \& Massagué, J. Balancing BMP signaling through integrated inputs into the Smad1 linker. Mol. Cell. 25, 441-54 (2007).

24. Alarcon, C. et al. Nuclear CDKs drive Smad transcriptional activation and turnover in BMP and TGF-beta pathways. Cell. 139, 757-769 (2008).

25. Chen, H. B., Shen, J., Ip, Y. T. \& Xu, L. Identification of phosphatases for Smad in the BMP/DPP pathway. Genes Dev. 20, 648-53 (2006)

26. Knockaert, M., Sapkota, G., Alarcón, C., Massagué, J. \& Brivanlou, A. H. Unique players in the BMP pathway: small C-terminal domain phosphatases dephosphorylate Smad1 to attenuate BMP signaling. Proc. Natl. Acad. Sci. U S A 103, 11940-5 (2006).

27. Duan, X., Liang, Y. Y., Feng, X. H. \& Lin, X. Protein serine/threonine phosphatase PPM1A dephosphorylates Smad1 in the bone morphogenetic protein signaling pathway. J. Biol. Chem. 281, 36526-32 (2006).

28. Eivers, E. et al. Mad is required for Wingless signaling and segment patterning in Drosophila and Xenopus. PLoS One 4, e6543 (2009).

29. Peifer, M., Pai, L. M. \& Casey, M. Phosphorylation of the Drosophila adherens junction protein Armadillo: roles for wingless signal and zeste-white 3 kinase. Dev. Biol. 166, 543-56 (1994).

30. Kirkpatrick, H., Johnson, K. \& Laughon, A. Repression of dpp targets by binding of brinker to mad sites. J. Biol. Chem. 276, 18216-18222 (2001).

31. Grieder, N. C., de Cuevas, M. \& Spradling, A. C. The fusome organizes the microtubule network during oocyte differentiation in Drosophila. Development. 127, 4253-4264 (2000)

32. Mazzalupo, S. \& Cooley, L. Illuminating the role of caspases during Drosophila oogenesis. Cell Death Differ. 13, 1950-1959 (2006).

33. Xu, M., Kirov, N. \& Rushlow, C. Peak levels of BMP in the Drosophila embryo control target genes by a feed-forward mechanism. Development. 1321, 637-47 (2005).

34. Siegfried, E., Chou, T. B. \& Perrimon, N. Wingless signaling acts through zeste white 3, the drosophila homolog of glycogen synthase kinase-3, to regulate engrailed and establish cell fate. Cell. 71, 1167-1179 (1992).

35. de Celis, J. F., Barrio, R. \& Kafatos, F. C. A gene complex acting downstream of dpp in Drosophila wing morphogenesis. Nature. 381, 421-424 (1996).

36. Grimm, S. \& Pflugfelder, G. O. Control of the gene optomotor-blind in Drosophila wing development by decapentaplegic and wingless. Science. 271 1601-4 (1996).

37. Kuroda, H., Fuentealba, L., Ikeda, A., Reversade, B. \& De Robertis, E. M. Default neural induction: neuralization of dissociated Xenopus cells is mediated by Ras/ MAPK activation. Genes Dev. 19, 1022-1027 (2005).

38. Eivers, E., Demagny, H., Choi, R. \& DeRobertis, E. M. Phosphorylation of Mad control competition between wingless and BMP signaling. Sci. Sig. 194, ra68 (2011).

39. Quijano, J. C., Stinchfield, M. J. \& Newfeld, S. J. Wg signaling via Zw3 and mad restricts self-renewal of sensory organ precursor cells in Drosophila. Genetics 189 809-24 (2011).

40. Brower, D. L. Engrailed gene expression in Drosophila imaginal discs. EMBO J. 5, 2649-2656 (1986).

41. Clemens, J. C. et al. Use of double stranded RNA interference in Drosophila cell lines to dissect signal transduction pathways. Proc. Natl. Acad. Sci. USA. 97, 6499-6503 (2000). 


\section{Acknowledgments}

We thank C. H. Heldin E. Laufer, Developmental Studies Hybridoma Bank and the Bloomington Stock Center for reagents. A.A. and M.R. were supported by the National Institutes of Health GM061331. M.J and D.L were supported by the National Science Foundation HRD-1302873 and the office of the California State University Chancellor (LSAMP). M. J. was a 2013 CSUPERB president's commission scholar. This research was funded by the National Institutes of Health SC3 GM103699 (E.E.).

\section{Author contributions}

A.A., M.R., M.J., D.L., A.C. and E.E. performed the research and analyzed the data. A.A. and E.E. designed the experiments, prepared the figures and wrote the manuscript.

\section{Additional information}

Supplementary information accompanies this paper at http://www.nature.com/ scientificreports

Competing financial interests: The authors declare no competing financial interests.

How to cite this article: Aleman, A. et al. Mad linker phosphorylations control the intensity and range of the BMP-activity gradient in developing Drosophila tissues. Sci. Rep. 4, 6927; DOI:10.1038/srep06927 (2014).

(1) (-) This work is licensed under a Creative Commons Attribution-NonCommercialNoDerivs 4.0 International License. The images or other third party material in this article are included in the article's Creative Commons license, unless indicated otherwise in the credit line; if the material is not included under the Creative Commons license, users will need to obtain permission from the license holder in order to reproduce the material. To view a copy of this license, visit http:// creativecommons.org/licenses/by-nc-nd/4.0/ 